\title{
Establishment of EBV latency in nasopharyngeal tumor epithelial cells by in vivo cell-mediated transfer infection
}

\author{
Fenggang $\mathrm{Yu}^{1 *}$, Yanan $\mathrm{Lu}^{1}$, Joshua K Tay ${ }^{1}$, Hironori Yoshiyama ${ }^{2 *}$ and Kwok Seng Loh ${ }^{1,3}$ \\ ${ }^{1}$ Department of Otolaryngology-Head and Neck Surgery, Yong Loo Lin School of Medicine, National University of Singapore, Singapore \\ ${ }^{2}$ Department of Microbiology, Shimane University Faculty of Medicine, Japan \\ ${ }^{3}$ Department of Otolaryngology-Head and Neck Surgery, National University Health System, Singapore
}

\begin{abstract}
Epstein-Barr virus (EBV) is a herpesvirus associated with approximately 1\% of tumors worldwide. Although EBV is consistently detected in nasopharyngeal carcinoma(NPC) biopsy, it is hardly detected in normal nasopharyngeal epithelium. The mechanism how virus establishes latent infection in tumor epithelial cells, including the source of virus and the route of entry, has not been fully elucidated largely due to the lack of appropriate in vivo models. We herein aim to investigate the potential route that epithelial cells are infected with EBV. To this end, we established in vivo model system by injection of cell-free EBV or EBV producer line Akata cells together with EBV negative NPC line HONE-1 cells. Akin to in vitro infections, we presented the first in vivo evidence that cell-mediated transfer infection via Akata cells was much more efficient than cell-free virus. These cells then expressed the EBV latency-associated small RNA EBERs, but not lytic antigens, such as BZLF1. However, when cells were inoculated at separate sites, EBV producer line Akata cell failed to demonstrate the ability of migrating from distant location to interact with HONE-1 cell to establish latent infection. In conclusion, cell-cell contact is critical for in vivo EBV infection of nasopharyngeal epithelial cells.
\end{abstract}

\section{Introduction}

Epstein-Barr virus (EBV) is a ubiquitous human herpesvirus infecting more than $90 \%$ of the adult human population. As the first human oncogenic virus identified, it is well known to be associated with malignancies of both lymphoid and epithelial origins such as Burkkit's lymphoma, gastric cancer, and nasopharyngeal carcinoma (NPC) $[1,2]$.

Following primary infection, EBV colonizes the memory B cell pool and establishes lifelong persistence as a latent infection [35]. According to the germinal center model [6], EBV preferentially establishes persistent infection within memory B cells in Waldeyer's ring, a ringed arrangement of lymphoid tissue in the pharynx. The virus then colonizes the peripheral lymphoid system, at a low level, by trafficking with these memory B cells as they circulate through the body and back to Waldeyer's ring. At Waldeyer's ring, these memory B cells receive unknown stimuli causing lytic reactivation, releasing virions back into the saliva through the oropharynx epithelium $[7,8]$.

NPC is a cancer arising in the epithelial lining of the nasopharynx, an anatomical region located just above the oropharynx. EBV DNA is consistently detected in undifferentiated NPC (WHO Type III) biopsies [9], but is rarely found in normal nasopharyngeal epithelial tissues $[10,11]$. Screening programs targeting healthy high-risk individuals seropositive for EBV IgA antibodies have also failed to demonstrate EBV infection in non-malignant nasopharyngeal biopsies [12]. However, EBV can be found in dysplastic preinvasive nasopharyngeal epithelial tissues [13], suggesting that EBV infection is an early event in oncogenesis. The clonal nature of EBV DNA in NPC [14] also supports EBV infection as an early event.

Although the association of NPC with EBV has been firmly established, the timing and site of EBV involvement during tumor development have been explained by circumstantial evidences and remain controversial. The source of EBV which delivers virus to nasopharyngeal epithelium remains uncertain and has not been addressed by existing epithelial infection models $[13,15]$. On the other hand, virus shedding into the saliva is a hallmark of persistent EBV infection and can be found also in healthy carriers [8]. It is thus possible that the source of EBV is from cell-free virus in the saliva, or alternatively, from memory B cells present in circulation or the submucosa of the nasopharynx. In vitro, EBV infection of telomerase-immortalized nasopharyngeal epithelial cell lines has been demonstrated with both cell-free virus and co-culture with EBV-producing Akata cells [16]. However, in vivo models have not been developed to validate these hypotheses. Thus, it is of great importance to evaluate EBV infection of nasopharyngeal epithelial cells in animal models.

In this study, we sought to address through an in vivo model whether EBV latency could be established in NPC tumor epithelial cells by directly co-injection with cell-free virus, or by co-injection with EBV producer cells that provide cell-mediated infection

\section{Materials and methods}

\section{Cell culture}

HONE-1 is an EBV negative epithelial tumor cell line derived from the biopsy specimen of an NPC patient [17]. The negativity of EBV

${ }^{\star}$ Correspondence to: Hironori Yoshiyama, Department of Microbiology, Shimane University Faculty of Medicine, Japan, Tel: +81 853-23-2111; E-mail: yosiyama@med.shimane-u.ac.jp

Fenggang Yu, Department of Otolaryngology-Head and Neck Surgery, Yong Loo Lin School of Medicine, National University of Singapore, Tel: (65) 6772 5309; Fax: (65) 6775 3820; E-mail: entyf@nus.edu.sg

Key words: epstein-Barr virus, nasopharyngeal carcinoma, latent infection, cellcell contact, co-injection animal models

Received: May 30, 2018; Accepted: June 16, 2018; Published: June 20, 2018 
infection in this cell line was confirmed by EBER in situ hybridization (ISH). EBV-producer line Akata, a human Burkitt's lymphoma-derived cell line carrying green fluorescent protein (GFP) tagged EBV episomes [18], was obtained from Prof. Kenzo Takada (Hokkaido University, Japan). Akata cells and HONE-1 cells were maintained in RPMI 1640 (Sigma, St. Louis, MO) with 10\% FBS.

\section{Virus production}

EBV producer line Akata cells were cross-linked with $100 \mu \mathrm{g} / \mathrm{ml}$ of human Fab immunoglobulin G (Jackson Immunoresearch, West Grove, $\mathrm{PA}, \mathrm{USA})$ for $24 \mathrm{~h}$ to induce lytic virus production. Cell suspensions were centrifuged for $5 \mathrm{~min}$ at $3,000 \mathrm{~g}$, and the supernatant was filtered through a $0.45-\mu \mathrm{m}$ filter to obtain cell-free virus. Titration of cell-free virus was done by real time PCR using the EBNA1 Taqman probe (Thermo Fisher Scientific, Waltham, MA USA). Using 10-fold serial dilutions of genomic DNA of Namalwa cells, which have two integrated copies of the EBV, a standard curve was constructed by plotting the logarithm of the EBV DNA copy number against the measured Ct values $(\mathrm{R} 2=0.9999)$. EBV genomes of DNase I treated Akata cell supernatant containing cell-free virus were quantified based on the standard curve generated. Aliquots of virus was stored at $-80^{\circ} \mathrm{C}$.

\section{In vitro $\mathrm{EBV}$ infection of $\mathrm{HONE}-1$ cells}

Two $\mathrm{ml}$ of supernatant containing cell-free virus at $7 \times 10^{7} \mathrm{DNA}$ copies $/ \mathrm{ml}$ was added to $2 \times 10^{5} \mathrm{HONE}-1$ cells in six-well plates in $2 \mathrm{ml}$ of medium. For infection through cell-to-cell contact, EBV producer Akata cells were stimulated overnight, washed, and resuspended in serum free media. Virus-producing Akata cells were added to six-well plates and incubated with $2 \times 10^{5} \mathrm{HONE}-1$ cells in a 1:1 ratio. Floating cells were removed at day 2 post infections, and GFP positive cells were counted on day 3 to calculate the infection efficiency.

\section{Animal models}

We tested our hypothesis in three in-vivo experimental models (Figure 1).

Co-injection Model: A mixture of $10^{6} \mathrm{HONE}-1$ cells and $10^{6} \mathrm{EBV}$ positive Akata cells were subcutaneously injected into first group of NOD/SCID/IL-2R $\gamma$ null (NSG) mice (4-5 wk old, $n=5$ ). In a second group of mice a mixture of $10^{6} \mathrm{HONE}-1$ and cell-free virus $\left(7 \times 10^{7} \mathrm{DNA}\right.$ copies/ml) was injected as a control.

Injection peripherally at separate sites: One million HONE-1 cells were subcutaneously injected into the front flank of NSG mice $(n=5)$. Seven to ten days post injection when tumors were palpable, $10^{6} \mathrm{EBV}$ producer Akata cells were subcutaneously injected into ipsilateral hind flank or contralateral front flank ( $\mathrm{n}=5 \mathrm{each})$.

Systemic injection of EBV-producer cells: One million HONE-1 cells were subcutaneously injected into the front flank $(n=5)$. Seven to ten days post-injection when tumors were palpable, $10^{6} \mathrm{EBV}$ producer Akata cells were intravenously delivered through the tail vein. To maximize the systemic interaction of HONE-1 and EBV-producer cells, we repeated these experiments by intramuscular injections of $10^{6}$ HONE-1 cells into the hind leg $(n=5)$, followed systemic delivery of $10^{6} \mathrm{EBV}$ producing Akata cells after 7-10 days. Finally, to recapitulate the invasive and metastatic behaviour of NPC, we used an orthotopic xenograft mouse model where HONE-1 cells were directly injected into the nasopharynx of mice $(n=5)$ [19]. Seven to ten days after nasopharyngeal injections, when an obvious decrease in body weight observed in mice, $10^{6} \mathrm{EBV}$ producer Akata cells were delivered through the tail vein. Two weeks after Akata cell administration, mice were sacrificed. Tumors were harvested, fixed in $10 \%$ formalin and processed into paraffin embedded tissue blocks. Sections of $4 \mu \mathrm{m}$ thickness were cut and used for histologic analysis. All the experiments were repeated twice.

\section{Histology and immunostaining}

$\mathrm{H}$ \& E staining, immunofluorescence (IF) staining, and immunohistochemistry (IHC) on tissue sections were conducted as previously described [20]. Chromogenic EBER in situ hybridization (EBER-ISH) was performed according to manual book (Dako, Glostrup, Denmark). For double staining, IHC of CK18 (Abcam, Cambridge, UK) was followed by EBER-ISH. EBER probe (Hs-EBER1, \#310271) for RNAscope was synthesized by Advanced Cell Diagnostics Inc. (Newwark, CA, USA). RNAscope in situ hybridization followed by IHC was performed according to the manufacture's protocol with some optimizations. Primary antibody CD79a were purchased from Abcam and Alexa Fluor secondary antibodies were from Thermo Fisher Scientific Inc. (Waltham, MA USA). Early phase lytic marker BZ1 antibody was purchased from Santa Cruz biotechnology Inc. (Dallas, Texas, USA). Working concentrations of antibodies were used at 1:100 to 1:200 dilutions.

\section{Statistics}

For the quantification of EBER ${ }^{+}$HONE-1 cells, xenograft tumors were cut through and every $50 \mu \mathrm{m}$ apart, sections of $4 \mu \mathrm{m}$ thickness were picked up for IHC analysis. Student's t-test was used for statistical analysis.

\section{Results}

\section{In vitro infection of HONE-1 cells}

The percentage of infected HONE-1 cells by cell-free virus was $1.02 \%$, in contrast that was $27.7 \%$ by cell-mediated infection with EBV producer line Akata cells (Figure 2). This suggests that infection through cell-cell contact is significantly more efficient than cell-free virus $(\mathrm{p}<0.05)$, consistent with previous studies $[21,22]$.

\section{In vivo infection of HONE-1 cells by co-injection}

The above results demonstrated that cell-cell contact is a more efficient way of infecting of HONE-1 cells. We then sought to investigate differences between infection through cell-mediated virus or infection through cell-free virus using in-vivo model. Ideally, normal primary nasopharyngeal epithelial cells would be used as a target for in-vivo infection, however, many difficulties in engrafting normal nasopharyngeal epithelial tissues in NSG mice still remain. Thus, EBVnegative HONE-1 cells was chosen as a target, because its xenografts are easily generated in vivo.

HONE-1 cells were injected together with either cell-free EBV virus or EBV-positive Akata cells into NSG mice subcutaneously (Figure 1). Tumor xenografts from both groups had a similar morphology, consisting of nests of malignant epithelial cells admixed with stromal cells and blood vessels (Figure $3 \mathrm{~A}$ and $\mathrm{B}$ ). We next checked for the presence of EBV in tumors using EBER-ISH. Patches of EBV positive cells were identified in tumors co-injected with Akata cells, but not in tumors co-injected with cell-free virus (Figure $3 \mathrm{C}$ and D).

To further clarify the identity of EBV infected cells, a combination of chromogenic EBER-ISH and IHC of cell lineage specific markers was employed. EBER-ISH has been considered as gold standard for 


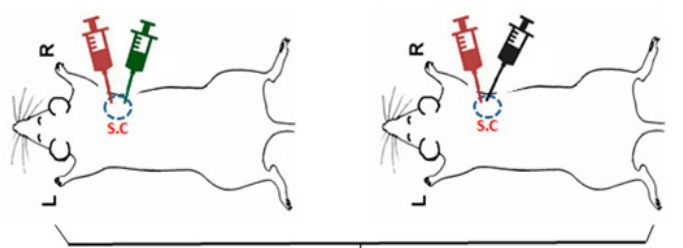

Co-Transplantation
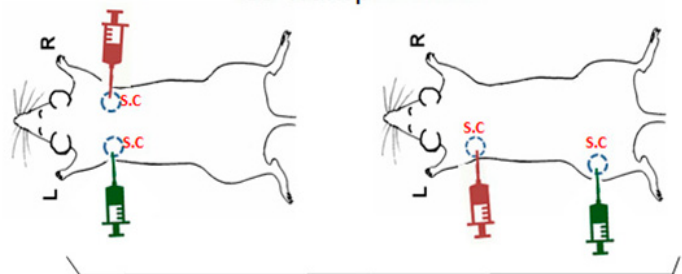

Peripheral administration of Akata cells
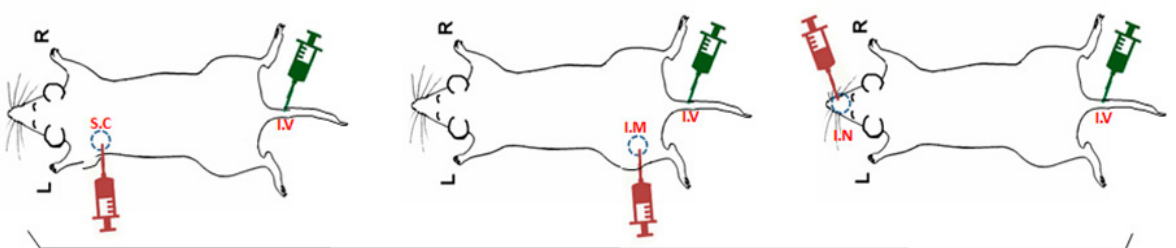

Systemic delivery of Akata cells through tail vein

Figure 1. Schematic diagram showing in vivo injection strategies. The top panel depicting co-injection scheme; the middle panel depicting separate injection scheme; the bottom panel depicting systematic delivery of Akata cells, while HONE-1 cells were administered via subcutaneous, muscular, and nasopharyngeal routes
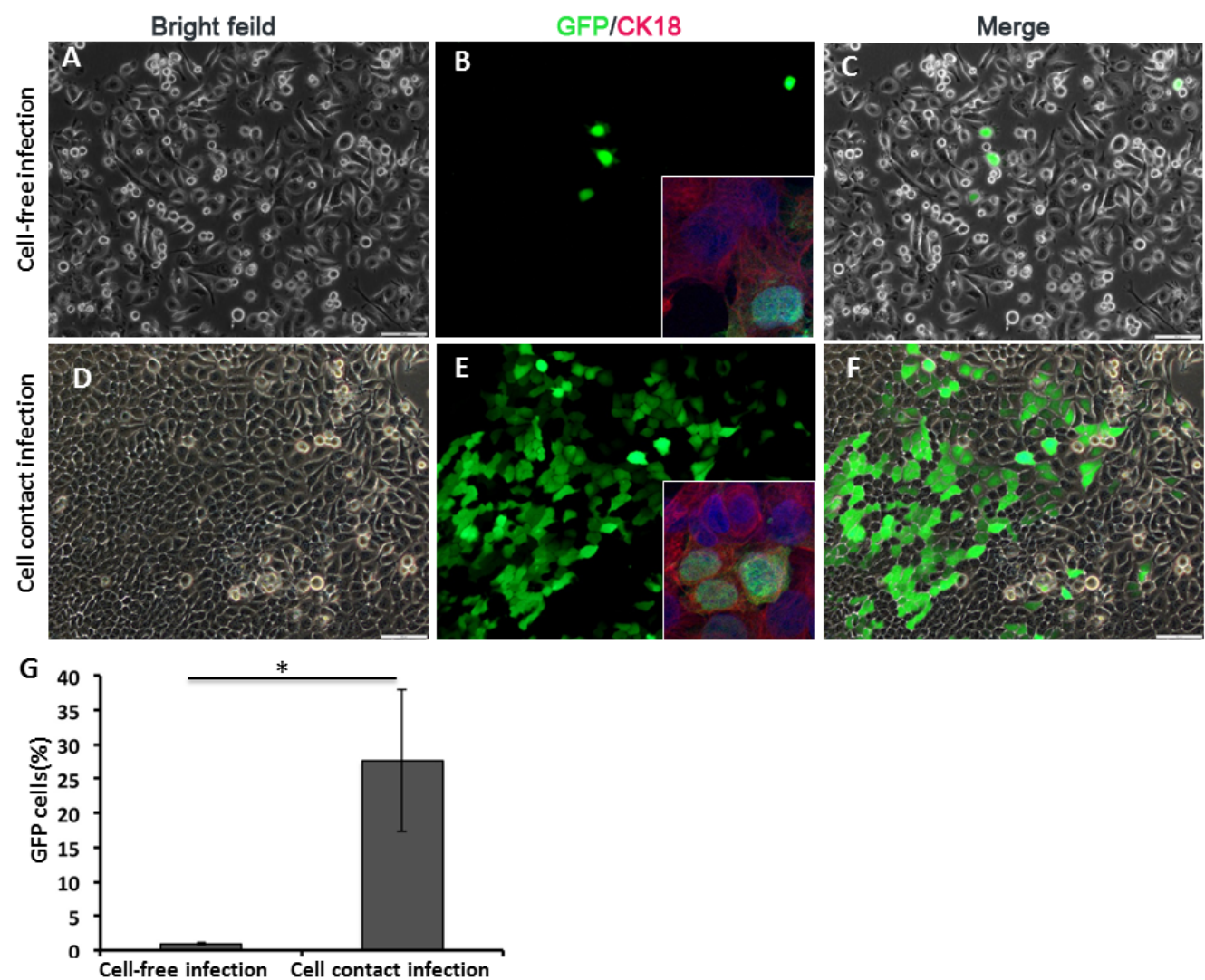

Figure 2. In vitro EBV infection of HONE-1 cells. HONE-1 cells were directly infected by cell-free EBV (A-C). HONE-1 cells were infected by cocultivation with Akata cells (D-F). The details of infection procedures are written in the materials and methods section. On day 3 post infection, the infection of HONE-1 cells was determined by GFP expression using fluorescent microscopy. Inserts confirm the epithelial identity of EBV infected cells by immunofluorescent staining of CK18 expression (in red). The nucleus was counterstained with DAPI. Scale bars $=100 \mu \mathrm{m}$. (G) The efficiencies of infection (mean of triplicates) were plotted and compared between the two modes of infection. (Student's $t$ test, $\mathrm{P}<0.05$ ). 


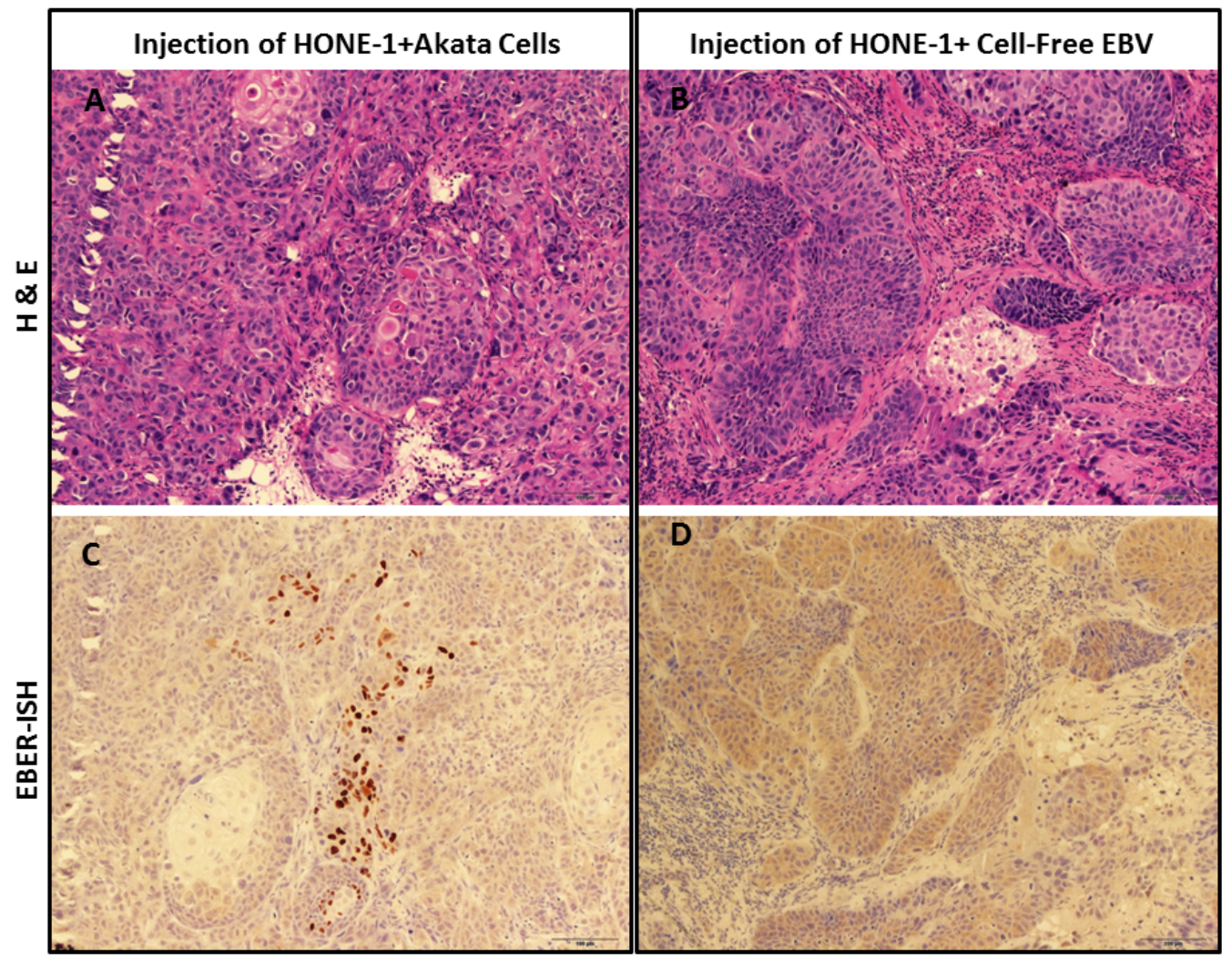

Figure 3. EBV transfer infection of HONE-1 cells by co-transplantation of Akata cells. NSG mice were transplanted with HONE-1 cells mixed with either cell-free EBV or EBV-producing Aktata cells, as described in the text. Four weeks post injection, the tumour xenografs were resected for histology analysis. H\&E staining and EBER in situ hybridization (ISH) were performed on adjacent sections at $4 \mu \mathrm{m}$ apart. The gross histology of tumour xenografts were shown by H\&E staining (A,B), and EBV infected cells were detected by conventional EBERISH $(C, D)$. EBER-positive cells (shown in brown) were found only in the group of co-injection with Akata cells (A,C), but not in cell-free virus co-injection group (B, D). Scale bars=100 $\mu \mathrm{m}$

detecting and localizing latent EBV in tissue samples. Of note, Ck18 is human antigen specific and able to distinguish human epithelial cells from murine epithelial cells. We did not identify any $\mathrm{EBER}^{+} \mathrm{CK}_{1} 8^{+}$cells in tumors of cell-free virus co-injection group (Figure 4A and B). In contrast, in the Akata co-injection group, except some EBER positive alone Akata cells, we detected co-expression of EBER and CK18 in a subset of cells, indicating successful EBV infection of HONE-1 cells (Figure 4C and D). To exclude the fusion of HONE-1 cells and Akata cells, double IF staining of CK18 and CD79a antibodies showed that HONE- 1 and Akata cells maintained a discrete distribution in the tumor (Figure 4E). Finally, we confirmed these findings with a combination of immunofluorescent staining and RNAscope, a fluorescence-based in situ hybridization technique with single transcript sensitivity. Colocalization of EBER RNA transcripts and CK18 was observed in a subgroup of HONE-1 cells (Figure 4F). Even with the high-resolution sensitivity, we were unable to detect any EBER ${ }^{+} \mathrm{HONE}-1$ cells in the cell-free virus injection group (Figure 4B).

Subsequently, IF staining was performed to examine the presence of viral lytic replication in EBV infected cells. None of the EBER ${ }^{+}, \mathrm{CK} 18^{+}$ HONE-1 cells expressed the early phase lytic marker BZ1, while about 2.0\% of Akata cells were positive for BZ1 (Figure $4 \mathrm{G}$ and $\mathrm{H}$ ), suggesting in HONE-1 cells latent infection was predominant. By quantifying the $\mathrm{EBER}^{+}, \mathrm{CK} 18^{+}$cells, we found the infection rate in HONE-1 cells to be approximately $0.2 \%$ when co-injected with Akata cells. Pre-Treatment of Akata cells with human IgG cross-linking prior to co-injection resulted in a modest increase in the infection rate to $0.3 \%$ (Figure $4 \mathrm{I}$ ).

\section{Infection of HONE-1 cells from peripheral or systemic EBV- producing Akata cells}

The peripheral injection model was aiming to mimic the interaction between nasopharyngeal epithelium and subepithelial lymphoid tissue. HONE-1 cells and Akata cells were administered subcutaneously into separate spots (Figure 1). Four weeks later, HONE-1 cells successfully formed xenograft tumor, whereas no tumor was observed at the site of Akata cell injection. Xenografted tumors were histologically analyzed, but no any EBER ${ }^{+}$cells could be detected by either conventional or RNAscope EBER-ISH (data not shown). This could be due to systematic interaction is not sufficient to attract Akata cells to the xenograft, as subcutaneous tumor usually remains encapsulated and contained at the site of injection. To maximize interaction within the body, HONE-1 cells were muscularly injected into hind limb, while Akata cells were administered subcutaneously. Indeed, in this way, tumor was very invasive and even spread to the bone. Again, any $\mathrm{EBV}^{+}$cells could not be found in the peripheral and tumor parenchyma (data not shown).

Systemic injection was intended to model the long-range interaction from peripheral circulation. In the first set of experiment, HONE-1 cells were subcutaneously or intramuscularly injected, while Akata cells were delivered via tail vein. Again, we were unable to detect any EBER ${ }^{+}$ cells in the tumor xenograft (Figure 5A and B). Finally, most relevant orthotopic model was employed. For this model, HONE-1 cells were directly injected into nasopharynx. Orthotopic injections resulted in weight loss, primary tumor formation in nasopharynx and metastases to lungs. Despite the high disease burden, we could not observe any 

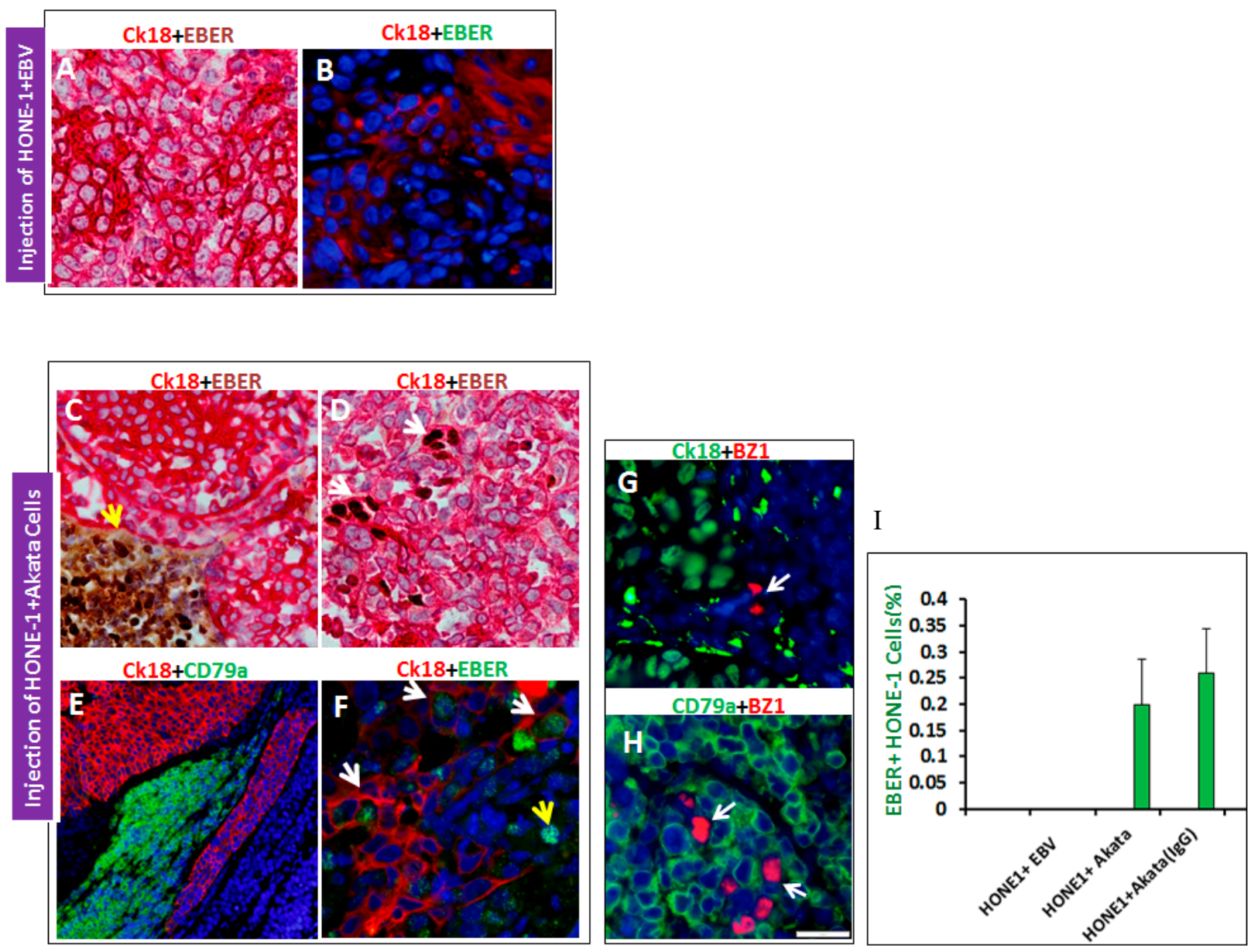

Figure 4. Confirmation of HONE-1 cells were subjected transfer-infection by Akata cells. No EBER positive cells were found in cell-free virus co-injection group no matter by conventional EBER-ISH. (A)or RNAScope EBER-ISH (B). Whereas in Akata cell co-injection group, two types of EBER positive cells were found, one type was CK18 negative (human antigen specific) and EBER positive Akata cells (C) and the other type was CK18 positive and EBER positive infected cells (D). Transplanted Akata cells (CD79a positive) and HONE-1 cells (CK18 positive) remain in discreet distributions, and no indication of cell fusion (E). (F): Fluorescent RNAscope EBER-ISH plus immunohistochemistry of CK18 strongly demonstrated a subset of HONE-1 cells (white arrow) were transfer infected by Akata cells (yellow arrow, devoid of CK18 staining). By EBV early phase lytic program marker BZ1 (in red), lytic infection was not detected in HONE-1 cells (G), but detected in Akata cells (H). Infection efficiency of HONE-1 cells was shown in (I): pre-treatment of Akata cells with human IgG can moderately enhance the infection rate
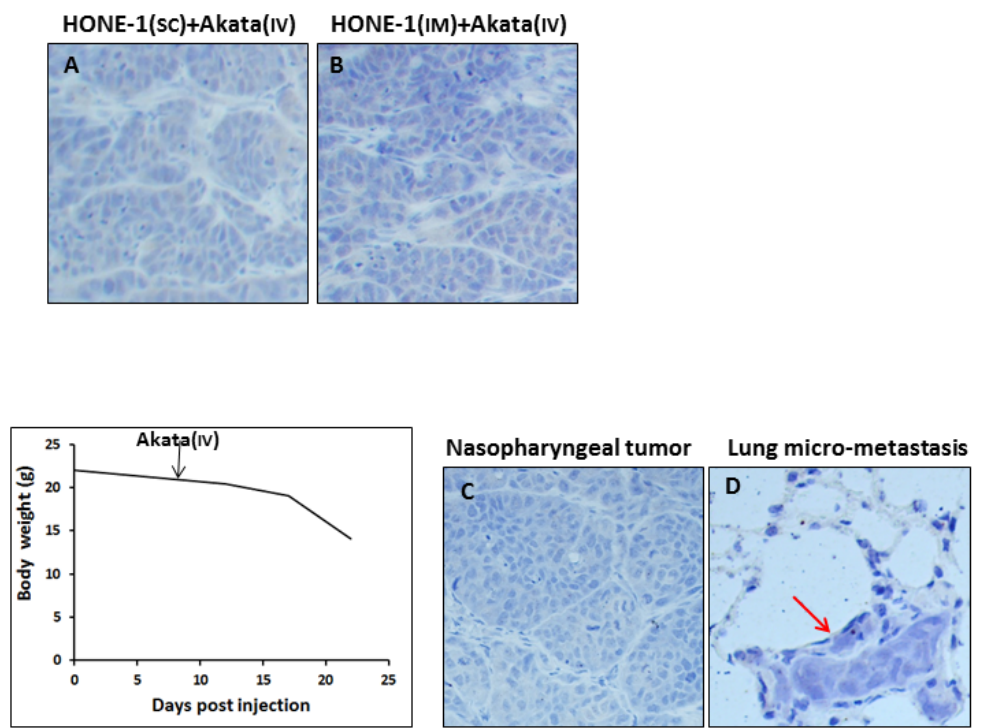

Figure 5. Systemic delivery of EBV producer Akata cells into HONE-1 xenograft. EBER-ISH showing EBER-positive cells was not detected in xenograft tumours resulted from subcutaneous (A) and intramuscular (B) models after intravenous injection of Akata cells, respectively. The orthotopic model recapitulates original cancer by showing the body loss (B), formation of primary tumour in nasopharynx $(\mathrm{C})$ and distant micro-metastasis in lung (D, shown by red arrow). None of EBER-positive cells was present in primary tumour (C) and distant metastasis (D) 
EBER $^{+}$cells by conventional and RNAscope EBER-ISH after systemic administration of Akata cells via tail vein (Figure 5C and D).

\section{Discussion and conclusion}

Our extensive in vivo animal models demonstrate that cell-mediated infection of EBV is more efficient than cell-free virus, extending the findings of previous in vitro studies [21,22]. This is also consistent with earlier findings that cell-free virus is usually incapable of or inefficient at infecting most epithelial cells in vitro [23]. The titer of cell-free virus used was high enough to achieve successful infection. We observed up to $60 \%$ of Daudi cells could be infected with the same cell-free virus preparation that failed to infect HONE-1 cells in vivo (data not shown). This indicates that susceptibility to infection, rather than viral titers, is the limiting factor. Although they are close in anatomy, cell-free EBV shed in the oral saliva encounters hurdles in infecting nasopharyngeal epithelium, such as the lack of the CD21 entry receptor in epithelial cells [24]. Although several EBV entry receptors into epithelium were discovered such as dimeric-IgA, $\beta 1$ integrin, Neuropilin, and Ephrin receptor A2, but none of them have been shown as efficient as CD21 [2528]. Earlier work by Shannon-Lowe et al. also demonstrated that EBV virions loading onto the surface of $\mathrm{B}$ cells were able to transfer more efficiently to CD21-negative epithelial cells in vitro, compared to cellfree virus [29]. Put together with our in vivo co-injection findings, we postulate that cell-mediated infection is the main mechanism by which EBV infection is established in nasopharyngeal epithelial cells. EBV infection of epithelial cells typically results in productive replication, as demonstrated in oral hairy leukoplakia [30] and in normal oral epithelium [31,32], while latent EBV infection usually occurs in epithelial malignancies [14]. Our results are also consistent with these facts, as we only observed latent infection in HONE-1 tumor xenograt. This is probably because genetic alterations are the prerequisite for permissive latent EBV replication, such as dysregulation of the cyclin D1 pathway and allelic deletion of chromosome 9p carrying the p16 locus in NPC tumor cells [13,33].

We performed peripheral and systemic injections of Akata cells to mimic infection by resident and circulating B lymphocytes. Even in orthotopic mice model with high tumor burden, we were unable to detect migration of Akata cells into HONE-1 tumors. Our conclusions in this regard are limited, because our experiments were performed with Akata lymphoma cells, but not with B lymphocytes which will migrate more actively [34]. Our model using the HONE-1 cell line also has limitations, because xenograft from HONE-1 cells is models the establishment of tumors but cannot mimic the pre-cancerous phenotype that probably releases chemokines to attract B cells [34].

Memory B cells latently infected with EBV do not always serve as the vehicle for transfer infection. Tugizov et al. [35], reported that EBV infected submucosal CD14+ monocytes migrated into the epithelium and expanded virus to oral epithelial cells to initiate productive viral infection within the terminally differentiated spinosum and granulosum layers. In their explant model, B lymphocytes played little part in the spread of EBV to keratinocytes, though B lymphocytes are the source of EBV. Consistent with this notion, important chemokine receptors such as CCR1 and CCR5, and chemotaxis to inflammatory chemokines were not detected in B lymphocytes [36-38]. Along similar line, Walling et al. [39], proposed another model of EBV transition from blood to oral epithelium in which EBV-infected Langerhans cell precursors serve to transport EBV to the oral epithelium as they migrate and differentiate into mucosa. EBV infection of monocytes, macrophages, and dendritic cells has been observed both in vitro and in vivo [40-42]. Detection of EBV-infected macrophages in healthy asymptomatic individuals suggests that, like circulating monocytes, tissue macrophages may also serve as a vehicle for virus transmission between the blood and epithelium compartments [40]. In conclusion, our model favors cellmediated transfer EBV infection via cell-cell contact rather than exposure to cell-free virus alone. Extending the concurrent results, we will pin-point which types of cells are the ultimate vehicles in the future study.

\section{Acknowledgements}

This work was supported by Yong Siew Yoon Research Grant from National University Cancer Institute, Singapore to KSL and Seed funding from National Medical Research Council, Singapore to FY. This work was supported by JSPS KAKENHI Grant Numbers JP18K07148 (HY) and Health Labor and Welfare, Japan (HY).

\section{References}

1. Thompson MP, Kurzrock R (2004) Epstein-Barr virus and cancer. Clin Cancer Res 10 803-821. [Crossref]

2. Yu F, Tan WJ, Lu Y, MacAry PA, Loh KS (2016) The other side of the coin: Leveraging Epstein-Barr virus in research and therapy. Oral Oncol 60: 112-117. [Crossref]

3. Babcock GJ, Decker LL, Volk M, Thorley-Lawson DA (1998) EBV persistence in memory B cells in vivo. Immunity 9: 395-404. [Crossref]

4. Babcock GJ, Decker LL, Freeman RB, Thorley-Lawson DA (1999) Epstein-Barr virusinfected resting memory B cells, not proliferating lymphoblasts, accumulate in the peripheral blood of immunosuppressed patients. J Exp Med 190: 567-576. [Crossref]

5. Thorley-Lawson DA (2001) Epstein-Barr virus: exploiting the immune system. Nat Rev Immunol 1: 75. [Crossref]

6. Laichalk LL, Hochberg D, Babcock GJ, Freeman RB, Thorley-Lawson DA (2002) The dispersal of mucosal memory B cells: evidence from persistent EBV infection. Immunity 16: 745-754. [Crossref]

7. Tugizov SM, Herrera R, Palefsky JM (2013) Epstein-Barr virus transcytosis through polarized oral epithelial cells. J Virol 87: 8179-8194. [Crossref]

8. Hadinoto V, Shapiro M, Sun CC, Thorley-Lawson DA (2009) The dynamics of EBV shedding implicate a central role for epithelial cells in amplifying viral output. PLoS Pathog 5: e1000496. [Crossref]

9. Andersson-Anvret M, Forsby N, Klein G, Henle W (1977) Relationship between the Epstein-Barr virus and undifferentiated nasopharyngeal carcinoma: Correlated nucleic acid hybridization and histopathological examination. Int J Cancer 20: 486-494.

10. Tao Q, Srivastava G, Chan AC, Chung LP, Loke SL, et al. (1995) Evidence for lytic infection by Epstein-Barr virus in mucosal lymphocytes instead of nasopharyngeal epithelial cells in normal individuals. J Med Virol 45: 71-77. [Crossref]

11. Sam C, Brooks L, Niedobitek G, Young L, Rickinson A, et al. (1993) Analysis of epstein-barr virus infection in nasopharyngeal biopsies from a group at high risk of nasopharyngeal carcinoma. Int J Cancer 53: 957-962.

12. Tay JK, Chan SH, Lim CM, Siow CH, Goh HL, et al. (2016) The Role of Epstein-Barr Virus DNA Load and Serology as Screening Tools for Nasopharyngeal Carcinoma. Otolaryngol Head Neck Surg 155: 274-280. [Crossref]

13. Tsang CM, Yip YL, Lo KW, Deng W, To KF, et al. (2012) Cyclin D1 overexpression supports stable EBV infection in nasopharyngeal epithelial cells. Proc Natl Acad Sci U $S$ A 109: E3473-E3482. [Crossref]

14. Pathmanathan R, Prasad U, Sadler R, Flynn K, Raab-Traub N (1995) Clonal proliferations of cells infected with Epstein-Barr virus in preinvasive lesions related to nasopharyngeal carcinoma. $N$ Engl J Med 333: 693-698. [Crossref]

15. Hutt-Fletcher LM (2016) The Long and Complicated Relationship between EpsteinBarr Virus and Epithelial Cells. J Virol 91. [Crossref]

16. Tsang, C. M., Zhang, G., Seto, E., Takada, K., Deng, W., Yip, Y. L., Man, C., Hau, P. M., Chen, H., and Cao, Y. Epstein-Barr virus infection in immortalized nasopharyngeal epithelial cells: Regulation of infection and phenotypic characterization. International journal of cancer. 2010,127, 1570-1583.

17. Yao K, Zhang HY, Zhu HC, Wang FX, Li GY, et al. (1990) Establishment and characterization of two epithelial tumor cell lines (hne-1 and hone-1) latently infected with epstein-barr virus and derived from nasopharyngeal carcinomas. Int $J$ Cancer 45 : 83-89. [Crossref] 
18. Kanda T, Yajima M, Ahsan N, Tanaka M, Takada K (2004) Production of high-titer Epstein-Barr virus recombinants derived from Akata cells by using a bacterial artificial chromosome system. J Virol 78: 7004-7015. [Crossref]

19. Smith PA, Merritt D, Barr L, Thorley-Lawson DA (2011) An orthotopic model of metastatic nasopharyngeal carcinoma and its application in elucidating a therapeutic target that inhibits metastasis. Genes Cancer 2: 1023-1033. [Crossref]

20. Yu F, Sim ACN, Li C, Li Y, Zhao X, et al. (2013) Identification of a subpopulation of nasopharyngeal carcinoma cells with cancer stem-like cell properties by high aldehyde dehydrogenase activity. Laryngoscope 123: 1903-1911.

21. Imai S, Nishikawa J, Takada K (1998) Cell-to-cell contact as an efficient mode of Epstein-Barr virus infection of diverse human epithelial cells. $J$ Virol 72: 4371-4378. [Crossref]

22. Chang Y, Tung CH, Huang YT, Lu J, Chen JY, et al. (1999) Requirement for cellto-cell contact in Epstein-Barr virus infection of nasopharyngeal carcinoma cells and keratinocytes. $J$ Virol 73: 8857-8866. [Crossref]

23. Pegtel DM, Middeldorp J, Thorley-Lawson DA (2004) Epstein-Barr virus infection in ex vivo tonsil epithelial cell cultures of asymptomatic carriers. J Virol 78: 1261312624. [Crossref]

24. Shannon-Lowe C, Rowe M (2011) Epstein-Barr virus infection of polarized epithelial cells via the basolateral surface by memory B cell-mediated transfer infection. PLoS Pathog 7: e1001338. [Crossref]

25. Chesnokova LS, Nishimura SL, Hutt-Fletcher LM (2009) Fusion of epithelial cells by Epstein-Barr virus proteins is triggered by binding of viral glycoproteins $\mathrm{gHgL}$ to integrins avß6 or avß8. Proc Natl Acad Sci U S A 106: 20464-20469. [Crossref]

26. Lin CT, Lin CR, Tan GK, Chen W, Dee AN, et al. (1997) The mechanism of Epstein-Barr virus infection in nasopharyngeal carcinoma cells. Am J Pathol 150: 1745-1756. [Crossref]

27. Wang HB, Zhang H, Zhang JP, Li Y, Zhao B, et al. (2015) Neuropilin 1 is an entry factor that promotes EBV infection of nasopharyngeal epithelial cells. Nat Commun 6: 6240 .

28. Chen J, Sathiyamoorthy K, Zhang X, Schaller S, White BEP, et al. (2018) Ephrin receptor A2 is a functional entry receptor for Epstein-Barr virus. Nat Microbiol 3: 172-180. [Crossref]

29. Shannon-Lowe C, Neuhierl B, Baldwin G, Rickinson A, Delecluse HJ (2006) Resting B cells as a transfer vehicle for Epstein-Barr virus infection of epithelial cells. Proc Natl Acad Sci U S A 103: 7065-7070. [Crossref]

30. Greenspan JS, Greenspan D, Lennette ET, Abrams DI, Conant MA, et al. (1985) Replication of Epstein-Barr virus within the epithelial cells of oral hairy leukoplakia, an AIDS-associated lesion. N Engl J Med 313: 1564-1571. [Crossref]
31. Frangou P, Buettner M, Niedobitek G (2005) Epstein-Barr virus (EBV) infection in epithelial cells in vivo: rare detection of EBV replication in tongue mucosa but not in salivary glands. J Infect Dis 191: 238-242. [Crossref]

32. Herrmann K, Frangou P, Middeldorp J, Niedobitek G (2002) Epstein-Barr virus replication in tongue epithelial cells. J Gen Virol 83: 2995-2998. [Crossref]

33. Lo KW, Huang DP, Lau KM (1995) p16 gene alterations in nasopharyngeal carcinoma Cancer Res 55: 2039-2043. [Crossref]

34. Kholodnyuk I, Rudevica Z, Leonciks A, Ehlin-Henriksson B, Kashuba E (2017) Expression of the chemokine receptors CCR1 and CCR2B is up-regulated in peripheral blood B cells upon EBV infection and in established lymphoblastoid cell lines. Virology 512: 1-7. [Crossref]

35. Tugizov S, Herrera R, Veluppillai P, Greenspan J, Greenspan D, et al. (2007) EpsteinBarr virus (EBV)-infected monocytes facilitate dissemination of EBV within the oral mucosal epithelium. J Virol 81: 5484-5496. [Crossref]

36. Wu L, Paxton WA, Kassam N, Ruffing N, Rottman JB, et al. (1997) CCR5 levels and expression pattern correlate with infectability by macrophage-tropic HIV-1, in vitro. $J$ Exp Med 185: 1681-1692. [Crossref]

37. Lee B, Sharron M, Montaner LJ, Weissman D, Doms RW (1999) Quantification of CD4, CCR5, and CXCR4 levels on lymphocyte subsets, dendritic cells, and differentially conditioned monocyte-derived macrophages. Proc Natl Acad Sci U S A 96: 5215-5220. [Crossref]

38. Brandes M, Legler DF, Spoerri B, Schaerli P, Moser B (2000) Activation-dependent modulation of B lymphocyte migration to chemokines. Int Immunol 12: 1285-1292. [Crossref]

39. Walling DM, Ray AJ, Nichols JE, Flaitz CM, Nichols CM (2007) Epstein-Barr virus infection of Langerhans cell precursors as a mechanism of oral epithelial entry, persistence, and reactivation. J Virol 81: 7249-7268. [Crossref]

40. Shimakage M, Kimura M, Yanoma S, Ibe M, Yokota S, et al. (1999) Expression of latent and replicative-infection genes of Epstein-Barr virus in macrophage. Arch Virol 144: 157-166. [Crossref]

41. Guerreiro-Cacais AO, Li L, Donati D, Bejarano MT, Morgan A, et al. (2004) Capacity of Epstein-Barr virus to infect monocytes and inhibit their development into dendritic cells is affected by the cell type supporting virus replication. J Gen Virol 85: 2767 2778. [Crossref]

42. Calattini S, Sereti I, Scheinberg P, Kimura H, Childs RW, et al. (2010) Detection of EBV genomes in plasmablasts/plasma cells and non-B cells in the blood of most patients with EBV lymphoproliferative disorders by using Immuno-FISH. Blood 116 : 4546-4559. [Crossref]

Copyright: (C2018 Yu F. This is an open-access article distributed under the terms of the Creative Commons Attribution License, which permits unrestricted use, distribution, and reproduction in any medium, provided the original author and source are credited. 